\title{
INTERVENCIONES PARA PREVENIR LA APARICIÓN DE SOBREPESO Y OBESIDAD EN NIÑOS MENORES DE CINCO AÑOS
}

\begin{abstract}
Catherine Bonilla ${ }^{1, a}$, Gisely Híjar ${ }^{1, b}$, Delia Márquez ${ }^{2, \mathrm{c}}$, Adolfo Aramburú1,d, Juan Pablo Aparco ${ }^{3, e}$, Ericson L. Gutiérrez ${ }^{1,4,}$ RESUMEN

La obesidad infantil representa uno de los mayores problemas de salud pública a nivel mundial. En el presente artículo se exponen las intervenciones para prevenir el sobrepeso y obesidad en niños menores de cinco años. Las intervenciones dirigidas a prevenir la obesidad en infantes fueron: promoción de la lactancia materna, vigilancia del crecimiento del niño y promoción de una adecuada alimentación complementaria, a través de la consejería nutricional con enfoque de alimentación responsiva, brindadas en distintos escenarios como el centro de salud o basados en el hogar a través de visitas domiciliarias. Las intervenciones dirigidas a prevenir obesidad en los preescolares incluyen las intervenciones con componentes de actividad física y nutrición que involucren activamente a los padres. La calidad de la evidencia de la mayoría de estudios es alta debido a que proviene de ensayos clínicos controlados, revisiones sistemáticas y metaanálisis. Todas estas intervenciones se realizan o podrían ser replicadas en nuestro país, mediante una adecuada contextualización.
\end{abstract}

Palabras clave: Obesidad pediátrica, Sobrepeso, Nutrición infantil, Programas y políticas de nutrición y alimentación. (fuente DeCS BIREME)

\section{INTERVENTIONS TO PREVENT THE DEVELOPMENT OF OVERWEIGHT AND OBESITY IN CHILDREN YOUNGER THAN FIVE YEARS}

\begin{abstract}
Childhood obesity is one of the most severe public health problems worldwide. The present study describes the interventions used to prevent overweight and obesity in children younger than 5 years. The objective of the interventions was to stimulate breastfeeding, monitor the child's growth, and promote adequate complementary feeding by means of nutritional counseling using a responsive feeding approach in different settings, including health centers and residences. The interventions included physical activity and nutritional counseling, with the active participation of the parents. The quality of evidence from most studies was high because the evidence was derived from controlled clinical trials, systematic reviews, and meta-analyses. All interventions were conducted or could be replicated in Peru by adequate contextualization.
\end{abstract}

Keywords: Pediatric obesity, Overweight, Infant nutrition, Nutrition programs and policies. (source: MeSH NLM)

\section{INTRODUCCIÓN}

El sobrepeso y la obesidad infantil representan un grave problema de salud pública tanto en países desarrollados como en países en desarrollo (1). La frecuencia de sobrepeso y obesidad en niños menores de cinco años, a nivel mundial, aumentó de 32 millones en la década de los noventa a 42 millones en el año 2013. En países de bajos y medianos ingresos la prevalencia de sobrepeso en preescolares supera el $30 \%{ }^{(2)}$.
En el Perú, según datos de la Encuesta Nacional de Hogares (ENAHO) 2013-2014, la prevalencia de exceso de peso (sobrepeso y obesidad) en niños menores de un año fue de $8,6 \%$; en niños de un año fue de $12,1 \%$; de dos años fue de $6,3 \%$, de tres años fue de $11 \%$ y de cuatro años fue de $8,1 \%{ }^{(3)}$. Además, este problema nutricional coexiste con la desnutrición y anemia infantil, los cuales aún no han sido resueltos en el Perú.

El sobrepeso y la obesidad infantil, si no son tratadas, tienden a mantenerse en la adolescencia y en la adultez ${ }^{(4)}$,

\footnotetext{
Centro Nacional de Salud Pública. Instituto Nacional de Salud. Lima, Perú.

Sección Posgrado. Facultad de Medicina. Universidad Nacional Mayor de San Marcos. Lima, Perú.

Centro Nacional de Alimentación y Nutrición. Instituto Nacional de Salud. Lima, Perú.

Facultad de Medicina. Universidad de San Martin de Porres. Lima, Perú.

a Nutricionista, magíster en Nutrición; ${ }^{\mathrm{b}}$ bióloga epidemióloga; ${ }^{\mathrm{c}}$ enfermera; ${ }^{\mathrm{d}}$ nutricionista, magíster en Salud Pública; ${ }^{\mathrm{e}}$ nutricionista; ${ }^{\mathrm{f}}$ médico, especialista en Gestión en Salud.

Recibido: 18/01/2017 Aprobado: 20/09/2017 En Línea: 29/11/2017
}

Citar como: Bonilla C, Híjar G, Márquez D, Aramburú A, Aparco JP, Gutiérrez EL. Intervenciones para prevenir la aparición de sobrepeso y obesidad en niños menores de cinco años. Rev Peru Med Exp Salud Publica. 2017;34(4):682-89. doi: 10.17843/rpmesp.2017.344.2636 
predisponiendo a las personas que lo padecen a desarrollar enfermedades como la diabetes, cardiopatías, trastornos osteomusculares, entre otros ${ }^{(5)}$. Estas enfermedades relacionadas a la nutrición son una de las principales causas de discapacidad y muerte, y los costos para los sistemas de salud, las empresas y las personas, son considerables y cada vez mayores.

Debido a las implicancias en salud pública, así como la falta de información a nivel local, se plantea el siguiente artículo que tiene como objetivo revisar la evidencia científica sobre intervenciones para prevenir la aparición de sobrepeso y obesidad en niños menores de cinco años.

\section{METODOLOGÍA}

Se realizó una búsqueda de la literatura hasta junio de 2017, utilizando las bases de datos Medline, Scielo y Cochrane Library. Se incluyeron artículos de metaanálisis, revisiones sistemáticas y ensayos controlados aleatorizados (ECA), que sean intervenciones para prevenir la aparición de sobrepeso y obesidad en niños menores de cinco años, publicadas en los últimos diez años, sin restricciones de idioma o ámbito geográfico.

Las estrategias de búsqueda se detallan a continuación:

\section{Medline:}

(((((“Obesity/prevention and control”[Mesh])) OR ("Overweight/prevention and control"[Mesh])) OR ("Pediatric Obesity/prevention and control"[Mesh]))) AND (("Child, Preschool"[Mesh]) OR "Infant"[Mesh]). La búsqueda fue filtrada para revisiones sistemáticas, ensayos aleatorizados controlados, publicados en los últimos 5 años.
Scielo:

prevención AND (obesidad OR sobrepeso) AND (niño)

\section{Cochrane:}

MeSH descriptor: [Weight Gain] explode all trees

MeSH descriptor: [Obesity] explode all trees

\#1 OR \#2

MeSH descriptor: [Diet] explode all trees

MeSH descriptor: [Exercise] explode all trees

MeSH descriptor: [Counseling] explode all trees

\#4 OR \#5 OR \#6

\#3 AND \#7

Filters: Cochrane Reviews

Luego de realizar la búsqueda de los artículos, estos fueron priorizados según la jerarquía de evidencia científica, de acuerdo a la "Directiva para los programas presupuestales en el marco de la programación y formulación del presupuesto del sector público para el año fiscal 2017", publicado en el Diario Oficial El Peruano en octubre de 2015 (Tabla 1).

\section{RESULTADOS}

\section{INTERVENCIONES PRENATALES}

Prevención de la ganancia excesiva de peso durante el embarazo

La evidencia actual indica que las intervenciones de prevención de ganancia excesiva de peso gestacional y de diabetes gestacional, reducen la macrosomía fetal, el cual es un factor que predispone a tener un mayor riesgo de obesidad a futuro ${ }^{(6)}$. Una reciente revisión Cochrane encontró que las intervenciones dirigidas a gestantes

Tabla 1. Intervenciones nutricionales específicas revisadas

\begin{tabular}{|c|c|}
\hline Intervenciones & Nivel de evidencia* \\
\hline \multicolumn{2}{|l|}{ Intervenciones prenatales } \\
\hline Intervenciones para prevenir la ganancia de peso excesivo durante el embarazo ${ }^{(6,7)}$ & $\mathrm{A} 1, \mathrm{~A} 2$ \\
\hline \multicolumn{2}{|l|}{ Intervenciones prevención de obesidad en infantes } \\
\hline Promoción de lactancia materna exclusiva y continuada ${ }^{(8,9)}$ & $\mathrm{A} 1, \mathrm{~A} 2$ \\
\hline Vigilancia del crecimiento saludable en la infancia ${ }^{(10,11)}$ & A2 \\
\hline Promoción de adecuada alimentación complementaria ${ }^{(12,13)}$ & A3 \\
\hline Consejería nutricional sobre prácticas de alimentación infantil (14,15) & A3 \\
\hline Intervenciones conductuales y no conductuales basadas en el hogar ${ }^{(16-20)}$ & $\mathrm{A} 2, \mathrm{~A} 3$ \\
\hline \multicolumn{2}{|l|}{ Intervenciones prevención de obesidad en preescolares } \\
\hline Intervenciones conductuales basadas en el hogar ${ }^{(21,22)}$ & A2 \\
\hline Involucramiento familiar $(23,24)$ & A2 \\
\hline \multicolumn{2}{|l|}{ Intervenciones en poblaciones rurales/minoritarias } \\
\hline Intervenciones comunitarias en poblaciones rurales/minoritarias ${ }^{(25)}$ & $\mathrm{A} 2$ \\
\hline
\end{tabular}


que incluyen dietas de bajo índice glicémico, ejercicios supervisados y no supervisados, o combinación de ambas, reducen el riesgo de ganancia excesiva de peso gestacional en $20 \%$, siendo el ejercicio supervisado la intervención de mayor efectividad $^{(7)}$ (Tabla 2 ).

\section{INTERVENCIONES PARA PREVENIR OBESIDAD EN INFANTES}

\section{Promoción de la lactancia materna exclusiva}

Existe una relación causal entre la lactancia materna y la prevención de obesidad infantil. La evidencia señala que existe una relación inversa entre la duración de lactancia materna exclusiva (LME) y el riesgo de obesidad. Los máximos beneficios se obtienen cuando la duración de la LME es mayor de seis meses y se prolonga hasta los dos años, apoyada con otros alimentos ${ }^{\left({ }^{(8)}\right.}$. Las mayores tasas de inicio temprano de lactancia materna, lactancia materna exclusiva y lactancia materna continuada, se observan cuando se brinda consejería o educación, tanto en casa como en la comunidad y a nivel del sistema de salud. La iniciativa de Hospitales Amigos de la Madre y el Niño es la intervención más efectiva para mejorar cualquier tipo de lactancia ${ }^{(9)}$. El objetivo de esta iniciativa es implementar los diez pasos para una lactancia exitosa que debe cumplir todos los servicios de maternidad y atención a los recién nacidos (Tabla 2).

\section{Vigilancia del crecimiento saludable en la infancia}

En la actualidad se aprecia una asociación entre el retraso del crecimiento y el aumento de la prevalencia de la obesidad en niños, tanto en países de bajos, medianos y altos ingresos $^{(10)}$, a la vez que existe evidencia que una ganancia acelerada de peso en la infancia incrementa el riesgo de marcadores de enfermedades crónicas no transmisibles. En ese sentido, la vigilancia de un crecimiento saludable, es decir, el monitoreo de los indicadores de crecimiento infantil para interpretar si el niño está creciendo normalmente o si presenta algún problema de crecimiento, constituye una estrategia importante para prevenir la obesidad infantil.

Durante el primer año de vida se presenta un aumento significativo del índice de masa corporal (IMC), seguido de un periodo de caída que finaliza entre los cuatro a seis años de edad, para ascender posteriormente hasta el final de la adolescencia. El incremento del IMC, justo después de alcanzar su valor más bajo alrededor de los seis años, es el que se conoce como rebote adiposo. Existe evidencia que, cuánto más tempranamente aparezca el rebote adiposo (principalmente antes de los cinco años), existe mayor riesgo de obesidad en edades posteriores. El niño con sobrepeso debe ser detectado tan pronto como sea posible para evitar la aparición de una obesidad mayor, por lo que es esencial seguir cuidadosamente la curva de IMC en todos los niños con especial atención a la edad del rebote adiposo temprano ${ }^{(11)}$.

\section{Promoción de una adecuada alimentación complementaria}

Alrededor de los seis meses de edad se debe iniciar la alimentación complementaria para cubrir las necesidades de energía y nutrientes del lactante, la cual debe brindarse junto con la lactancia materna. Esta alimentación complementaria debe aumentar gradualmente la consistencia y la variedad de todos los grupos alimentarios, evitando alimentos procesados y ultraprocesados.

El consumo de una inadecuada alimentación complementaria en la infancia tiene serias consecuencias en la composición corporal futura. Esto se ha observado en lactantes alimentados con fórmulas lácteas, alta en proteínas y baja en grasas, al presentar sobrepeso y obesidad ${ }^{(12)}$. Esta alta ingesta de proteínas (superior a la leche materna) está asociada con un crecimiento acelerado y con un rebote adiposo precoz, así como con incrementos en los factores de crecimiento ${ }^{(13)}$. Por lo que una estrategia relacionada sería brindar al infante una adecuada alimentación complementaria.

\section{Consejería nutricional sobre prácticas de alimentación infantil}

La consejería nutricional sobre prácticas de alimentación infantil que busca que los cuidadores alimenten a su niño adoptando una conducta responsiva, ha demostrado ser efectiva en prevenir la obesidad infantil.

Un estudio reciente examinó el efecto de una intervención de crianza responsiva que incluye la alimentación responsiva (prestar atención a las necesidades de alimentación del niño, mirándolo y respondiendo a los signos de hambre y saciedad) en la ganancia de peso infantil. La intervención estaba dirigida a madres primerizas a través de visitas domiciliarias, donde las enfermeras brindaron mensajes de alimentación infantil, higiene del sueño, juegos activos, regulación emocional y educación para el registro del crecimiento. Una acelerada ganancia de peso, asociada a posterior obesidad fueron menores entre los infantes del grupo de intervención comparados con el grupo control ${ }^{(14)}$.

La consejería nutricional sobre prácticas de alimentación infantil, brindada de manera temprana, en el paquete de atención integral brindado por los servicios de salud, también ha demostrado ser efectiva en prevenir la obesidad infantil. El proyecto NOURISH en Australia realizó la comparación entre la consejería temprana sobre prácticas de alimentación infantil versus la atención habitual de las madres en los servicios de salud. El proyecto brindó sesiones grupales a madres seleccionadas a cargo de nutricionistas y psicólogos, sobre alimentación y crecimiento saludables, y demostró ser efectiva en mejorar 
Tabla 2. Revisiones sistemáticas de intervenciones para prevenir la aparición de sobrepeso y obesidad en niños menores de cinco años

\begin{tabular}{|c|c|c|c|}
\hline Autor, año & $\begin{array}{l}\text { Tipo de } \\
\text { estudio }\end{array}$ & Intervención & Resultados \\
\hline \multicolumn{4}{|c|}{ Intervenciones para prevenir la ganancia excesiva de peso gestacional (PG) } \\
\hline $\begin{array}{l}\text { Muktabhant } \\
\text { B. et al } \\
2015^{(7)}\end{array}$ & $\begin{array}{l}\text { Metaanálisis } \\
\text { de } 65 \text { estudios } \\
\text { controlados } \\
\text { aleatorizados }\end{array}$ & $\begin{array}{l}\text { Intervenciones dietéticas (reducción de } \\
\text { calorías, azúcar o grasa) o ejercicio regular } \\
\text { (caminatas, danza o aeróbicos) o ambos. }\end{array}$ & $\begin{array}{l}\text { Reducción del riesgo de ganancia excesiva de PG (RR 0,80, } \\
\text { IC } 95 \% 0,73 \text { a } 0,87 \text { ). Dietas de bajo índice glicémico, ejercicios } \\
\text { supervisados o no, o combinación de dieta y ejercicios produjeron } \\
\text { similar efecto. }\end{array}$ \\
\hline
\end{tabular}

Promoción de la lactancia materna (LM) exclusiva y continuada

Aguilar et al. Revisión

sistemática de133 estudios

Lactancia materna (LM) para la prevención de sobrepeso y obesidad en la infancia

Revisión

Sinha et al. $2015^{(9)}$ sistemática y metaanálisis de 197 estudios
Intervenciones de consejería o educación para incrementar la frecuencia de lactancia materna (LM)

LM resulta eficaz en la prevención de la obesidad, aunque su duración sea breve. LM> 6 meses y prolongada hasta los dos años, apoyada con otros alimentos produce el máximo beneficio. La introducción de alimentos complementarios pasados los 6 meses de vida disminuye el riesgo de alergia alimentaria y puede prevenir la obesidad.

Consejería o educación producen mayor tasa de inicio temprano de LM exclusiva y continuada, sea que se brinden en casa o la comunidad (RR 1,85, IC 95\% 1,0 a 3,17), a través de los sistemas de salud y la comunidad (RR 2,52, IC $95 \%$ 1,39 a 4,59) o en cualquier escenario (RR 1,97, IC 95\% 1,74 a 2,24). La iniciativa Hospitales Amigos de la Madre y el Niño fue la intervención más efectiva para mejorar las tasas de LM exclusiva y continuada (RR $1,66, \mathrm{IC} 95 \% 1,34$ a 2,07$)$.

Consejeria nutricional sobre prácticas de alimentación infantil

El grupo de intervención recibió material educativo sobre alimentación, higiene del

Estudio

Savage et al. $2016^{(14)}$

controlado aleatorizado

sueño, juego social activo, cartilla educativa de del parto con visitas domiciliarias periódicas a cargo de enfermeras. El grupo control recibió una intervención sobre seguridad en el hogar apropiada para el desarrollo.

Grupo de intervención: sesiones quincenales interactivas de grupo en centros de salud a cargo de nutricionistas y psicólogos con aleatorizado
Estudio controlado

experiencia pediátrica, sobre alimentación saludable, relación entre alimentación y

$2012^{(15)}$

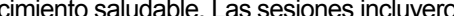
estrategias con enfoque cognitivo-conductual para mejorar la autoeficacia materna y construir un ambiente de apoyo.
El puntaje de ganancia de peso condicional medio fue menor en el grupo de intervención (-0,18, IC 95\% -0,36 a -0,001), reflejando una ganancia de peso más lenta en este grupo $(0,18$, IC $95 \% 0,02$ a $-0,34)$; este efecto no se relacionó con la modalidad de lactancia (predominantemente lactancia materna o no). Los infantes del grupo de intervención tuvieron menor percentil promedio de peso/ longitud al año de edad que los infantes en el grupo control $(57,5 \%$, IC $95 \% 52,56 \%$ a $62,37 \%$ vs $64,4 \%$, IC $95 \% 59,94 \%$ a $69,26 \%$, $\mathrm{p}=0,04)$ y menor probabilidad de presentar sobrepeso a la edad de 1 año $(5,5 \%$ vs. $12,7 \%, p=0,05)$.

Luego de 9 meses de seguimiento, los niños del grupo control tuvieron mayor puntaje $z$ de IMC para edad $(0,42 \pm 0,85$ vs 0,23 $\pm 0,93, p=0,009$ ) y mayor probabilidad de ganancia acelerada de peso desde la línea de basal (OR: 1,5, IC 95\% 1,1 a 2,1, p=0,014). Las madres del grupo control tuvieron mayor probabilidad de utilizar prácticas de alimentación no responsiva que no responden a señales de saciedad del niño, como fomentar la alimentación utilizando la comida como recompensa $(15 \%$ vs. $4 \%, p=0,001)$ o utilizando juegos $(67 \%$ vs. $29 \%$, p<0,001).

Intervenciones conductuales y no conductuales basadas en el hogar

Intervenciones conductuales:

a) intervenciones nutricionales $y / 0$ alimentación responsiva dirigidas a padres de infantes;

Redsell et al Revisión $2016^{(16)} \quad$ sistemática de 35 estudios b) promoción de lactancia materna y apoyo a

la lactancia para madres;

c) estilo de vida de los padres y la familia, a

través de visitas domiciliarias.

d) intervenciones en salud materna.

Intervenciones no conductuales: manipulación de la composición de la fórmula láctea.
Las intervenciones nutricionales y/o alimentación responsiva dirigidas a padres de infantes $(n=12)$ mejoraron prácticas de alimentación y tuvieron impacto en el peso del infante. La promoción de lactancia materna y apoyo a la lactancia para madres $(n=5)$ tuvo efecto positivo en la lactancia, pero no en el peso del infante. Intervenciones de estilos de vida de los padres y la familia $(n=4)$ tuvieron un impacto significativo en los hábitos de alimentación, pero menores mejoras que aquellas enfocadas en nutrición. Las intervenciones en salud materna $(n=3)$ tuvieron impacto en las prácticas de alimentación, pero no en el peso del infante.

Entre las intervenciones no conductuales, las fórmulas lácteas con proteína hidrolizada o reducida tuvieron efecto positivo sobre la reducción de peso.

Intervenciones dirigidas a mejorar la dieta y la capacidad de respuesta de los padres a las señales infantiles, mostraron ser más prometedoras en términos de cambio de comportamiento autorreportado. 


\begin{tabular}{|c|c|c|}
\hline $\begin{array}{l}\text { Ciampa et al. } \\
2010^{(17)}\end{array}$ & $\begin{array}{l}\text { Revisión } \\
\text { sistemática de } \\
10 \text { estudios }\end{array}$ & $\begin{array}{l}\text { Intervenciones diseñadas para prevenir o } \\
\text { reducir el sobrepeso y la obesidad en niños } \\
\text { menores de } 2 \text { años. }\end{array}$ \\
\hline
\end{tabular}

Hesketh et al. Revisión $2010^{(18)}$ sistemática de 23 estudios infantil, promoción de alimentación saludable, actividad física y/o reducción de actividades sedentarias en infantes y niños de 0 a 5 años.
Estrategias de prevención de obesidad
Wen et al. $2012^{(19)}$
Estudio controlado aleatorizado
Promoción de lactancia materna, adecuada introducción de alimentos sólidos, juegos activos, nutrición y actividad física familiar con seguimiento periódico por personal de salud.
Estudio de seguimiento longitudinal Wen et al. 2015 (20) de estudios controlados aleatorios
Seguimiento longitudinal 3 años después de una intervención basada en visitas domiciliarias periódicas de personal de salud dirigidas al control del sobrepeso infantil.
Los lugares de estudio fueron el hogar $(n=2)$, hospitales $(n=3)$, aulas de clase $(n=4)$, o una combinación $(n=1)$. La duración de las intervenciones fue en general menor de 6 meses y tuvieron éxito moderado en modificar indicadores como ingesta dietética, actitudes y conocimientos de nutrición de los padres. Ninguna intervención mejoró el peso del infante.

La mayoría de estudios fueron en centros preescolares o guarderías $(n=9)$, o en el hogar $(n=8)$. Aproximadamente, la mitad dirigidos a niños de bajo nivel socioeconómico $(n=12)$. La mayoría de estudios reportados en centros preescolares o guarderías no mostraron evidencia de efectos en la modificación de hábitos que contribuyan a la obesidad.

Índice de masa corporal (IMC) promedio más bajo en el grupo de intervención $(16,53)$ que en el grupo control $(16,82),(p=0,04)$. Los niños en el grupo de intervención tuvieron mayor probabilidad de comer una o más porciones de vegetales ( $89 \%$ vs. $83 \%, p=0,03$ ) y menor probabilidad de recibir alimentos como recompensa $(62 \%$ vs $72 \%, p=0,03$ ) respecto al grupo control. El porcentaje de niños que cenaban mirando TV, o que veían TV durante las comidas fue significativamente más bajo en el grupo de intervención que en el grupo control ( $56 \%$ vs. $68 \%, p=0,01$; y $66 \%$ vs. $76 \%, p=0,02$, respectivamente)

Las diferencias entre los grupos de intervención y control a los 2 años de edad en el IMC y z score IMC desaparecieron con el tiempo. A la edad de 5 años, la diferencia media en IMC fue de 0,03 (IC 95\% -0,30 a 0,37). No se detectaron efectos de la intervención temprana en hábitos alimentarios, calidad de vida, actividad física y tiempo de ver TV, a la edad de 5 años.

Intervenciones conductuales basadas en el hogar
Ling et al.

$2016^{(21)}$

Revisión sistemática de

24 estudios

Intervenciones de actividad física medida objetivamente en niños de 2 a 5 años.
Todas las intervenciones que alcanzaron efecto significativo en la actividad física objetivamente medida, fueron desarrolladas en centros de cuidado infantil e incluyeron un componente de actividad física estructurado, 6 incluyeron componentes múltiples, 5 integraron teorías o modelos, y 4 incluyeron activamente a los padres. El diseño de los estudios, las mediciones de actividad física y las intervenciones fueron heterogéneas, dificultando llegar a conclusiones definitivas.
Intervenciones para reducir la obesidad y

Revisión mejorar los hábitos obesogénicos, incluyendo Sisson et al. sistemática de actividad física, dieta y tiempo frente a $2016^{(22)}$

97 estudios
La mayoría de intervenciones tuvieron efectos sobre la reducción de la obesidad $48 \%(n=14)$, aumento de la actividad física $73 \%$ $(n=30)$, mejora de la dieta $87 \%(n=399$, y disminución del tiempo frente a pantallas $63 \%(n=5)$.

\section{Involucramiento familiar}

Barr-

Anderson et al. $2013^{(23)}$

Revisión sistemática de 27 estudios pantallas, en niños de 3 a 5 años de centros de cuidado diurno.
Involucramiento familiar en las intervenciones de prevención o tratamiento de obesidad en niñas afroamericanas. $\begin{array}{lll}\text { Golley et al. } & \text { Revisión } & \text { Intervenciones dirigidas a padres para } \\ 2011^{(24)} & \text { mejorar el peso y los patrones dietéticos y/o }\end{array}$ $\begin{array}{ccc}2011^{(24)} & \text { sistemática de } & \text { de actividad física de sus hijos, así como el } \\ 25 \text { estudios } & \text { contenido de la intervención y las técnicas de }\end{array}$ cambio de comportamiento utilizadas.

En todos los estudios, los efectos sobre los comportamientos relacionados con el peso fueron prometedores, pero a menudo no significativos.

Las intervenciones efectivas estuvieron dirigidas a reducir la ingesta y densidad energética, y elegir alimentos saludables. Las intervenciones que fueron consideradas efectivas tuvieron similares características: padres responsables de la participación e implementación, mayor involucramiento de los padres e inclusión de identificación de barreras, reestructuración del entorno del hogar, autocontrol, técnicas específicas de fijación de metas de cambio de comportamiento.

Intervenciones comunitarias en poblaciones rurales/minoritarias

Intervenciones en el hogar: visitas domiciliarias intensivas durante el embarazo hasta el nacimiento o en la infancia temprana intervenciones en centros de atención primaria Laws et al Revisión de salud y durante la atención médica general. $2014^{(25)}$ sistemática de 32 estudios
Intervenciones en centros preescolares.

Intervenciones en la comunidad: programas de promoción de la salud federales y preparación para la escuela para familias de bajos ingresos e intervenciones comunitarias para familias latinas con niños de edad preescolar.
Las intervenciones que empezaron en la infancia (antes de los dos años) tuvieron un impacto positivo en los comportamientos relacionados con obesidad (por ejm. Calidad de la dieta), pero pocos midieron el impacto a largo plazo en la ganancia de peso saludable. Los hallazgos entre los preescolares (3-5 años) fueron mixtos, con las intervenciones más exitosas incluyendo altos niveles de involucramiento familiar, uso de técnicas de cambio de comportamiento, un enfoque de desarrollo de habilidades y enlaces a recursos de la comunidad. 
las prácticas de alimentación responsiva y para prevenir ganancias aceleradas de peso en infantes en el primer año de vida ${ }^{(15)}$, sin mantener este último efecto después del segundo año de vida, seis meses después de terminar la intervención (Tabla 2).

Intervenciones conductuales y no conductuales basadas en el hogar

Una reciente revisión sistemática estudió todas las intervenciones basadas en el hogar para prevenir la obesidad en el infante y las clasificó en conductuales y no conductuales. Entre las intervenciones conductuales para prevenir la obesidad infantil durante los primeros 1000 días de vida, se encuentran las intervenciones nutricionales y/o alimentación responsiva dirigidas a los padres, la promoción de la lactancia materna y el apoyo a la lactancia a madres, adecuados estilos de vida de los padres y la familia, y las intervenciones en salud materna, mientras que las intervenciones no conductuales incluyen aquellas que modifican la composición de las fórmulas lácteas.

La evidencia indica que todas las intervenciones son efectivas en mejorar las prácticas de alimentación y nutrición infantil, mientras tanto, las intervenciones nutricionales y/o de alimentación responsiva, como aquellas que modifican las fórmulas lácteas con proteína hidrolizada o reducida, tienen, además efectos positivos en el peso del infante, es decir, previenen una ganancia excesiva de peso ${ }^{(16)}$. Otras revisiones sobre intervenciones conductuales dirigidos a infantes, realizadas en el decenio pasado y enfocadas en solo una intervención, como educación nutricional ${ }^{(17)}$ o en actividad física en entornos educativos ${ }^{(18)}$, no reportaron mejoras en el peso de niños menores de dos años, aunque sí efectos positivos en los hábitos que contribuían a prevenirla (Tabla 2).

Las intervenciones tempranas basadas en el hogar son efectivas en prevenir una ganancia excesiva de peso en infantes, al menos durante el tiempo de exposición a la intervención. En Australia, el programa Healthy Beginnings Trial, buscó promover la lactancia materna, la adecuada introducción de alimentos sólidos, juegos activos, así como nutrición y actividad física familiar, a través de visitas domiciliarias de enfermeras capacitadas, y demostró ser efectiva en prevenir la ganancia excesiva de peso infantil y en mejorar prácticas de alimentación saludable y de actividad física durante los tres años de intervención ${ }^{(19)}$, desapareciendo las diferencias entre intervenidos y controles durante la segunda fase de seguimiento sin ninguna intervención, cuando los niños alcanzaron la edad de cinco años ${ }^{(20)}$.

\section{INTERVENCIONES PARA PREVENIR OBESIDAD EN PREESCOLARES}

\section{Intervenciones conductuales basadas en el hogar}

En el caso de las intervenciones dirigidas a prevenir obesidad en preescolares, la evidencia actual indica que las más efectivas incluyen componentes de actividad física y nutrición, involucran activamente a los padres y están basadas en teorías conductuales (modificación de conductas), además, utilizan la educación interactiva como la estrategia más común dirigida a los padres, y la educación interactiva junto con experiencias prácticas, dirigida a los niños ${ }^{(21)}$. Estas mismas intervenciones dirigidas a prevenir obesidad infantil también son efectivas al implementarse en centros de cuidado diurno ${ }^{(22)}$ (Tabla 2).

\section{Involucramiento familiar}

El involucramiento familiar o de los padres en las intervenciones para la prevención de obesidad infantil ha sido previamente estudiado. Cuando se realizan estrategias de cambio de comportamiento, tiene sentido involucrar a la familia, debido a que el niño no tiene el control de las decisiones de estilo de vida saludable.

La evidencia al respecto señala que, al parecer, esta intervención por sí sola puede presentar resultados insuficientes ${ }^{(23)}$, a pesar de ser un factor que contribuye de manera importante en las intervenciones efectivas ${ }^{(24)}$.

\section{INTERVENCIONES PARA PREVENIR OBESIDAD EN INFANTES DE POBLACIONES RURALES}

\section{Intervenciones comunitarias}

Las poblaciones económicamente desfavorecidas o indígenas en el mundo también presentan incrementos en sus tasas de sobrepeso/obesidad. En ese sentido, es necesario identificar las intervenciones para prevenir la obesidad en los primeros años de vida de estas poblaciones, y reducir la correspondiente carga de desarrollar enfermedades crónicas a futuro. La evidencia actual muestra que las intervenciones dirigidas a estas poblaciones son conducidas en el hogar, a través de visitas domiciliarias intensivas; centros de atención primaria, dentro de programas dirigidos a la salud materno-infantil; centros preescolares, o en la comunidad, a través de programas de promoción de la salud y preparación para la escuela. Las intervenciones que empezaron en la infancia, antes de los dos años, tienen impacto positivo en la calidad de la dieta, mientras que en los preescolares (tres a cinco años de edad) son efectivas cuando incluyen altos niveles de involucramiento familiar, utilizan técnicas de cambio de comportamiento, con enfoque de desarrollo de habilidades y se enlazan con recursos y las preferencias alimentarias de la comunidad ${ }^{(25)}$ (Tabla 2).

\section{LIMITACIONES DEL ESTUDIO}

Este trabajo no fue una revisión sistemática de la información relacionada al tema, debido a que solo se 
incluyeron metaanálisis, revisiones sistemáticas y ensayos controlados aleatorizados, de los que se dispusiera del texto completo. No se realizó búsqueda de literatura gris. A pesar de ello, los artículos encontrados brindan evidencia sobre las principales intervenciones mencionadas.

\section{CONCLUSIONES}

Las intervenciones efectivas dirigidas a prevenir sobrepeso y obesidad en el infante incluyen: promoción de lactancia materna, vigilancia del crecimiento del niño, promoción de alimentación complementaria adecuada, a través de la consejería nutricional con enfoque de alimentación responsiva, brindadas en distintos escenarios como el centro de salud o basados en el hogar a través de visitas domiciliarias. Las intervenciones dirigidas a prevenir obesidad en los preescolares, incluyen las intervenciones con componente conductuales que incorporen actividad física y alimentación saludable y que involucren activamente a los padres.

$\mathrm{Si}$ bien las intervenciones presentadas provienen de estudios publicados en otros países, se debe considerar que la mayoría se basa en asegurar un adecuado crecimiento en los primeros años de vida y promocionar adecuados hábitos de alimentación y actividad física, intervenciones que bien podrían ser replicadas en nuestro país.

La promoción de la lactancia materna, la vigilancia del crecimiento del niño y la consejería nutricional, son intervenciones que ya se vienen realizando en la atención integral del niño, por lo que se cuenta con oportunidades para enfatizar en el seguimiento de aquellos niños en riesgo de presentar obesidad infantil y promover cambios conductuales.

Todas las conductas saludables a ser incorporadas en el infante deben ser sostenidas en el tiempo y apoyadas por sus familiares más cercanos, padres y/o tutores, quienes son responsables de su alimentación y convertir cualquier cambio de conducta en un hábito de vida con el objetivo de lograr una modificación favorable y significativa en su salud actual y futura. Un tipo de alimentación es considerado como saludable no solo cuando restringe el mayor aporte calórico, sino que, además, incluye aspectos como tipo de alimentos, combinaciones en la dieta, horarios de ingesta y aporte calórico, todos estos aspectos personalizados según la actividad diaria de cada infante. Estas intervenciones, a su vez, tendrán proyección en todas las etapas de vida.

Específicamente, en el caso de las poblaciones vulnerables (económicamente desfavorecidas 0 indígenas), la evidencia indica que la efectividad de las intervenciones se incrementa cuando incluyen enfoques de desarrollo de habilidades y se incorporan con los recursos y las preferencias alimentarias de la comunidad. Al presentar nuestro país una gran variedad cultural y grandes brechas económicas y sociales, sería importante considerar estos aspectos en la elaboración de programas dirigidos a estas comunidades.

Finalmente, considerando que aún persiste el problema de desnutrición y anemia en nuestra población infantil, es importante que las actuales políticas y programas de salud orientadas a enfrentar estos problemas, sean integradas, con el fin de que la ayuda alimentaria que se brinde no tenga efectos indeseados, como incremento de los niveles de sobrepeso y obesidad.

Contribuciones de autoría: CB, GH, ELG, participaron en la concepción del trabajo, todos los autores participaron en la redacción del manuscrito, en la revisión crítica del manuscrito y en la aprobación final del manuscrito.

Fuente de financiamiento: estudio financiado por el Instituto Nacional de Salud de Perú.

Conflicto de intereses: los autores declaran no tener conflictos de interés en relación a los contenidos de este documento.

\section{REFERENCIAS BIBLIOGRÁFICAS}

1. Estrategia mundial sobre régimen alimentario, actividad física y salud. Informe de una consulta de la OMS sobre Sobrepeso y obesidad infantiles [Internet]. Ginebra: Organización Mundial de la Salud; 2017 [Citado el 1 de enero de 2017] Disponible en: http://www.who. int/dietphysicalactivity/childhood/es/

2. Comisión para acabar con la obesidad infantil. Informe de una consulta de la OMS sobre Datos y cifras sobre obesidad infantil [Internet]. Ginebra: Organización Mundial de la Salud;
2017 [Citado el 1 de enero de 2017] Disponible en: http://www.who.int/ end-childhood-obesity/facts/es/

3. Instituto Nacional de Salud. Informe técnico: estado nutricional por etapas de vida en la población peruana; 2013-2014. [Internet]. Lima: Instituto Nacional de Salud; 2015 [Citado el 1 de enero de 2017]. Disponible en: http://www.portal. ins.gob.pe/es/component/rsfiles/preview ?path=cenan $\% 252 \mathrm{FV}$ igilancia\%2Bde $\% 2$ BIndicadores\%2BNutricionales\%2BB\%2 52FVIN\%2BENAHO\%2BPOBLACI ON\%2B2013-2014\%2B220116.pdf
4. Albañil Ballesteros MR, Rogero Blanco ME, Sánchez Martín M, Olivas Domínguez A, Rabanal Basalo A, Sanz Bayona MT. Riesgo de mantener obesidad desde la infancia hasta el final de la adolescencia. Rev Pediatr Aten Primaria 2011;13(50):199-211.

5. Consenso sobre factores de riesgo de enfermedad cardiovascular. Obesidad. Arch Argent Pediatr. 2005;103(3):262-81.

6. Schellong K, Schulz S, Harder T, Plagemann A. Birth weight and long-term overweight risk: systematic review and a meta-analysis including 643,902 persons 
from 66 studies and 26 countries globally. PLoS One. 2012;7(10):e47776. doi: 10.1371/journal.pone.0047776.

7. Muktabhant B, Lawrie TA, Lumbiganon P, Laopaiboon M. Diet or exercise, or both, for preventing excessive weight gain in pregnancy. Cochrane Database Syst Rev. 2015; (6):CD007145. doi: 10.1002/14651858.CD007145.pub3.

8. Aguilar Cordero MJ, Sánchez López AM, Madrid Baños N, Mur Villar N, Expósito RuizM,Hermoso RodríguezE. Breastfeeding for the prevention of overweight and obesity in children and teenagers; systematic review. Nutr Hosp. 2014;31(2):606-20. doi: 10.3305/nh.2015.31.2.8458.

9. Sinha B, Chowdhury R, Sankar MJ, MartinesJ, Taneja S, Mazumder S, et al.Interventions to improve breastfeeding outcomes: a systematic review and meta-analysis. Acta Paediatr. 2015;104(467):114-34. doi: 10.1111/apa.13127.

10. Abdullah A. The Double Burden of Undernutrition and Overnutrition in Developing Countries: an Update. Curr Obes Rep. 2015;4(3):337-49. doi: 10.1007/ s13679-015-0170-y.

11. Négre V. Early assessment of overweight for preventing obesity. Rev Prat. 2015; 65(10):1264-9.

12. Rolland-Cachera MF, Akrout M, Péneau S. Nutrient Intakes in Early Life and Risk of Obesity. Int J Environ Res Public Health. 2016;13(6). pii: E564. doi: 10.3390/ijerph13060564.

13. Socha P, Grote V, Gruszfeld D, Janas R, Demmelmair H, Closa-Monasterolo R. et al. Milk protein intake, the metabolic-endocrine response, and growth in infancy: data from a randomized clinical trial. Am J Clin Nutr. 2011;94(6 Suppl):1776S-1784S. doi: 10.3945/ ajcn.110.000596.

14. Savage JS, Birch LL, Marini M, Anzman-Frasca S, Paul IM. Effect of the INSIGHT Responsive Parenting Inter- vention on Rapid Infant Weight Gain and Overweight Status at Age 1 Year: A Randomized Clinical Trial. JAMA Pediatr. 2016;170(8):742-9. doi: 10.1001/ jamapediatrics.2016.0445.

15. Daniels LA, Mallan KM, Battistutta D, Nicholson JM, Perry R, Magarey A. Evaluation of an intervention to promote protective infant feeding practices to prevent childhood obesity: outcomes of the NOURISH RCT at 14 months of age and 6 months post the first of two intervention modules. Int J Obes (Lond). 2012;36(10):1292-8. doi: 10.1038/ ijo.2012.96.

16. Redsell SA, Edmonds B, Swift JA, Siriwardena AN, Weng S, Nathan D, et al. Systematic review of randomised controlled trials of interventions that aim to reduce the risk, either directly or indirectly, of overweight and obesity in infancy and early childhood. Matern Child Nutr. 2016;12(1):24-38. doi: 10.1111/ mon.12184.

17. Ciampa PJ, Kumar D, Barkin SL, Sanders LM, Yin HS, Perrin EM, et al. Interventions aimed at decreasing obesity in children younger than 2 years: a systematic review. Arch Pediatr Adolesc Med. 2010;164(12):1098-104. doi: 10.1001/ archpediatrics.2010.232.

18. Hesketh KD, Campbell KJ. Interventions to prevent obesity in 0-5 year olds: an updated systematic review of the literature. Obesity (Silver Spring). 2010;18 Suppl 1:S27-35. doi: 10.1038/oby.2009.429.

19. Wen LM, Baur LA, Simpson JM, Rissel C, Wardle K, Flood VM. Effectiveness of home based early intervention on children's BMI at age 2: randomised controlled trial. BMJ. 2012;344:e3732. doi: 10.1136/bmj.e3732.

20. Wen LM, Baur LA, Simpson JM, Xu H, Hayes AJ, Hardy LL, et al. Sustainability of Effects of an Early Childhood Obesity Prevention Trial Over Time:
A Further 3-Year Follow-up of the Healthy Beginnings Trial. JAMA Pediatr. 2015;169(6):543-51. doi: 10.1001/jamapediatrics.2015.0258.

21. Ling J, Robbins LB, Wen F. Interventions to prevent and manage overweight or obesity in preschool children: A systematic review. Int J Nurs Stud. 2016;53:270-89. doi: 10.1016/j.jinurstu.2015.10.017.

22. Sisson SB, Krampe M, Anundson K, Casthe $S$. Obesity prevention and obesogenic behavior interventions in child care: A systematic review. Prev Med. 2016;87:57-69. doi: 10.1016/j.ypmed.2016.02.016.

23. Barr-Anderson DJ, Adams-Wynn AW, DiSantis KI, Kumanyika S. Family-focused physical activity, diet and obesity interventions in African-American girls: a systematic review. Obes Rev. 2013;14(1):29-51. doi: 10.1111/j.1467-789X.2012.01043.x.

24. Golley RK, Hendrie GA, Slater A, Corsini N. Interventions that involve parents to improve children's weight-related nutrition intake and activity patterns - what nutrition and activity targets and behaviour change techniques are associated with intervention effectiveness? Obes Rev. 2011;12(2):114-30. doi: 10.1111/j.1467789X.2010.00745.x.

25. Laws R, Campbell KJ, van der Pligt P, Russell G, Ball K, Lynch J, et al. The impact of interventions to prevent obesity or improve obesity related behaviours in children (0-5 years) from socioeconomically disadvantaged and/or indigenous families: a systematic review. BMC Public Health. 2014;14:779. doi: 10.1186/14712458-14-779.

Correspondencia: Ericson Gutiérrez Dirección: Calle Cápac Yupanqui 1400. Jesús María. Lima 11, Perú Teléfono: (511) 748-1111 anexo 2113 Correoelectrónico:eringunza@yahoo.es 